Valparaiso University

ValpoScholar

Nursing and Health Professions Faculty

Publications

Nursing and Health Professions Faculty

2019

The effect of a perioperative nursing elective on nursing career

paths

Nola A. Schmidt

Janet M. Brown

Follow this and additional works at: https://scholar.valpo.edu/nursing_fac_pubs

Part of the Nursing Commons

This Article is brought to you for free and open access by the Nursing and Health Professions Faculty at ValpoScholar. It has been accepted for inclusion in Nursing and Health Professions Faculty Publications by an authorized administrator of ValpoScholar. For more information, please contact a ValpoScholar staff member at scholar@valpo.edu. 


\section{Yesterday's standard:}

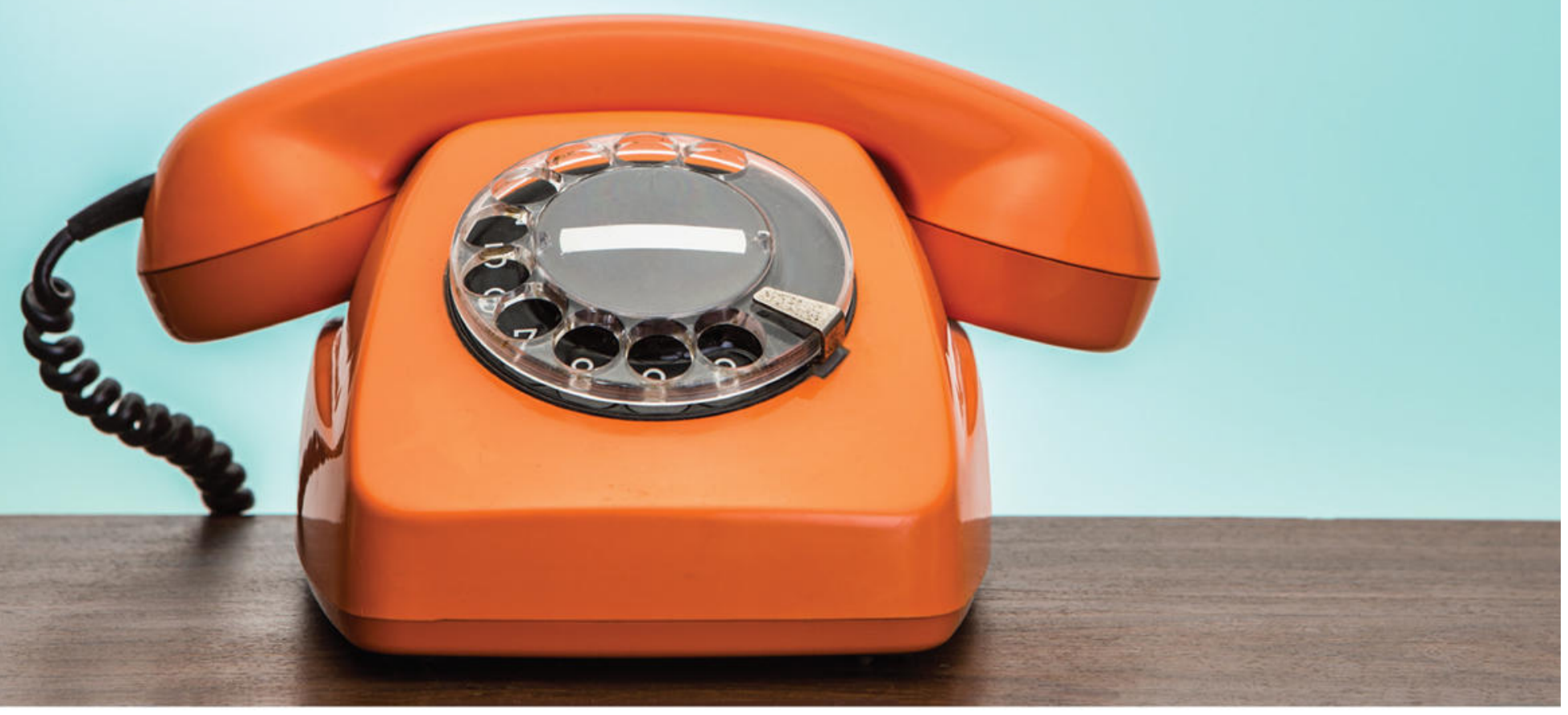

Nice if you like collectibles, not if you like efficiency.

Yesterday's best isn't good enough for today's demanding OR. In fact, when you compare Vaporized Hydrogen Peroxide Sterilizers, you'll see the truth. V-PRO ${ }^{\oplus}$ Sterilizers are validated on more than 43 device materials compared to just 22 for STERRAD..$^{*}$ Outperforming STERRAD Sterilizers in every important category, V-PRO Sterilizers deliver with faster cycles and greater weight and chamber capacity, so you'll process more trays per shift. Today's one and only true choice is V-PRO. Talk to your STERIS representative today or visit steris.com/vpro.

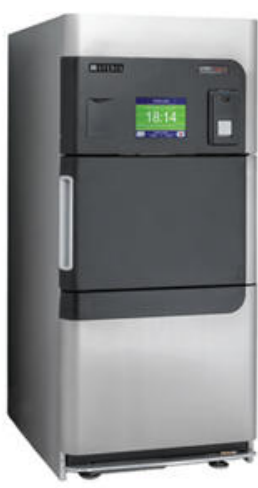




\title{
The Effect of a Perioperative Nursing Elective on Nursing Career Paths
}

\author{
Nola A. Schmidt, PhD, RN, CNE; Janet M. Brown, PhD, RN
}

\begin{abstract}
Nurse educators play a role in addressing the growing perioperative nurse shortage in the United States by implementing strategies to entice new graduates to this specialty. The purpose of our study was to determine if an undergraduate perioperative nursing elective influenced the career choices of nurses four to nine years after they graduated from a baccalaureate nursing program in the midwestern United States. Using a descriptive study design, 23 of 50 nurses responded to a survey about positions they have held since graduating and the influence of a perioperative nursing elective on their career choices. Twenty-six percent of nurses in this sample went on to work in the perioperative specialty, and the majority indicated they continued to consider perioperative nursing as a career choice. Considering the potential long-term effects of this strategy, incorporating a perioperative nursing elective into nursing school curricula could be helpful to address the shortage of perioperative nurses.
\end{abstract}

Key words: career choice, perioperative nursing elective, perioperative course, nursing shortage, undergraduate nursing program.

istorically, perioperative nursing experiences have been excluded from most United States nursing programs. ${ }^{1,2}$ Students are unlikely to select perioperative nursing as a career specialty because of a deficit of perioperative content in nursing school curricula. ${ }^{3}$ This lack of exposure is concerning because in $2014,55.8 \%$ of perioperative nurse leaders reported having difficulty recruiting staff members and $67.9 \%$ reported that they expected to encounter recruitment problems in the next five years. ${ }^{4}$ This shortage may affect patient safety and access to surgical care. In addition, hospitals and surgical centers may lose revenue if adequate staffing is unavailable. ${ }^{5}$

As a result of the minimal exposure to perioperative nursing, students in traditional undergraduate nursing programs fail to gain an appreciation for the specialty. ${ }^{6}$ Organizations such as AORN have advocated for the development of elective courses that contain perioperative content $t^{1,2,7}$ based on the assumption that such experiences will entice students to select perioperative nursing as a career choice after graduation. However, we found limited research to support the idea that students' career choices are influenced by immersion in perioperative content. The purpose of our study was to determine if an undergraduate perioperative nursing elective influenced career choices of nurses four to nine years after graduating from nursing school.

\section{LITERATURE REVIEW}

Most research about the inclusion of perioperative content in undergraduate nursing programs focuses on courses and orientations. Findings are mixed; some approaches only show limited success in influencing students to choose perioperative nursing immediately after graduation.

Tschirch et $\mathrm{al}^{8}$ made perhaps the most radical attempt at introducing perioperative nursing to the curriculum by requiring a perioperative nursing course as the first clinical course in a new baccalaureate program. The researchers based the content for the course on AORN's Periop 101. Through a practice partnership with a local health system, students (sample size not reported) participated in a six-day 
rotation through settings that included the preoperative screening area, OR, postanesthesia care unit (PACU), outpatient day surgery area, and endoscopy suite. A preceptor education program helped to provide a thorough orientation to 21 hospital preceptors. Clinical instructors evaluated students using verbal and written feedback. All of the students were capable of meeting the clinical objectives set for the course. The students' and preceptors' responses to the course were positive. Some anecdotal reporting indicated that two of the students reported working in a perioperative setting after graduation, while another, who worked in an intensive care unit, reported that the experience could be translated to critical care. With the exception of Tschirch et al, ${ }^{8}$ most perioperative content has been incorporated into nursing programs by adding experiences to existing courses and developing electives.

\section{Perioperative Content Added to Existing Courses}

One way of introducing perioperative nursing to undergraduate students is to incorporate perioperative content into courses that are already part of the curriculum. Resources for integrating perioperative nursing experiences into existing courses can be found in the literature. ${ }^{9}$ Mott ${ }^{10}$ integrated a 64-hour perioperative clinical experience into a senior-level critical care course for seven baccalaureate nursing students who had an interest in perioperative nursing. After an orientation to the OR, students rotated through perioperative areas and observed presentations about perioperative content. Although no questions were asked about the influence of the course on their career choices, one comment from a student indicated that she would consider perioperative nursing as a career. Overall, the feedback about the experience was positive, but some students indicated they were disappointed with the lack of opportunities to perform skills.

\section{One way of introducing perioperative nursing} to undergraduate students is to incorporate perioperative content into courses that are already part of the curriculum.

In response to a local shortage of perioperative nurses, Chappy et $\mathrm{al}^{11}$ created a new perioperative option as part of an existing senior-level nursing course. Students spent 10 weeks in either a mental health or acute care setting followed by a four-week clinical rotation working with a preceptor in their chosen setting. The researchers offered students the alternative option of an immersive 14-week perioperative clinical experience. Those who selected the immersion option completed Periop 101 in addition to their required course content. For the first 10 weeks, students spent 12 hours with a nurse preceptor. In the final four weeks, students completed a minimum of 126 hours by working alongside their preceptors. Some students were immediately hired into perioperative nursing positions upon graduation by the organizations where they completed their rotations. Penprase et al ${ }^{12}$ implemented a similar strategy and found that in two semesters, all students $(N=18)$ were offered positions in the perioperative setting, and 14 students accepted the offers.

Castelluccio ${ }^{13}$ developed a two-hour orientation to ease students' anxieties about observing in the OR, encourage their interest in the specialty, and explain the different roles in the perioperative setting. The students learned about aseptic technique and practiced skills such as donning surgical garb, opening sterile supplies, and scrubbing for procedures. Because of the orientation, some students requested extra days in the perioperative area. The orientation generated more student interest in externships and senior practicums in perioperative nursing.

\section{Perioperative Nursing Electives}

Gregory et $\mathrm{a}^{14}$ worked in partnership with a local hospital to address a local shortage in perioperative nursing by developing a 1.5-credit course that was offered during the summer between the students' junior and senior years. The course included a nurse externship program offered by the hospital, an orientation, 180 hours of clinical experiences, and evidence-based practice projects. Because of student interest, a second course was added to provide more extensive content, which resulted in the participation of 120 students in four years. The partnership reduced the hospital's perioperative nursing shortage and the need for agency nurses.

Messina et $\mathrm{al}^{15}$ organized a 15 -week practicum for seven seniors during the last semester of an undergraduate nursing program. Students indicated that they found the experience worthwhile, although some expressed that it was not what they had anticipated. Most students shared negative perceptions of their experiences, including less time with 
patients, a lack of opportunities to perform skills, and the rigid structure of the perioperative environment. Two of the seven students who completed the practicum asked to remain in the OR for their senior capstone clinicals. These students found that by working in the OR, they improved their communication skills and benefited from the structured environment. They also stated that they enjoyed using technology and felt rewarded by providing support and education to their patients. Both of these students were hired by the hospital to work in the OR after graduation.

Foran ${ }^{16}$ used a mixed-methods design to study Australian nursing students' perceptions about how guided practical experiences in the perioperative area during their education program influenced their desire to seek future employment in the OR. She found that approximately $30 \%$ of the students who had a guided experience (ie, clinical experience under the supervision of a proficient perioperative nurse) considered working in the OR, compared with approximately $18 \%$ of students who had a nonguided perioperative experience. In a follow-up analysis, Foran ${ }^{17}$ reported that students with a guided experience in the perioperative area had a $76 \%$ pass rate on a multiple-choice assessment that measured surgical knowledge. The pass rate for students who had either a nonguided perioperative experience or no perioperative experience was only $56 \%$.

\section{Studying the Effect of a Perioperative Nursing Elective}

We conducted an initial qualitative study ${ }^{5}$ to determine student perceptions about the influence of a perioperative nursing elective on career preferences. Nineteen students who completed a three-credit course that included both lecture and clinical components participated in one of two focus groups. We identified four themes. The first was "Opening their eyes: career advantages." Students began to consider perioperative nursing as a possible career choice for themselves. They were able to articulate positive aspects of the position, such as teamwork and how long perioperative nurses remained in their positions because they liked the specialty. The second theme, "Being captive: career disadvantages," captured students' negative perceptions about the specialty. Students expressed concerns about feeling confined in the environment and believed that there were limited opportunities for advancement. "Paradox unrecognized" was the third theme. Students often reported conflicting opinions. For example, although longevity in the perioperative area was viewed positively as a sign of job satisfaction, students also believed longevity would seem monotonous and career limiting. The final theme was "I'll be a better nurse." Students, including those who did not want to pursue a position in perioperative nursing, valued their experiences and recognized how they will be better prepared to care for patients wherever they work. Although the course stimulated some interest in perioperative nursing, most students were not persuaded to select this specialty as an immediate career. Many students indicated that, because of the experience, they were more open to considering perioperative nursing in the future.

In the literature, there are other instances illustrating the success of elective perioperative courses on the recruitment of new graduates directly into the perioperative setting. For example, Holmes ${ }^{18}$ showed that $25 \%$ of students who took a perioperative nursing elective went on to work in either the PACU or the OR, and that $20 \%$ enrolled in nurse anesthetist programs. Additionally, Ball et al ${ }^{7}$ piloted a perioperative experience that eventually became a regularly offered elective course. After graduation, two of the four students who completed the pilot were hired by the partnering hospital.

\section{In the literature, there are other instances illustrating the success of elective perioperative courses on the recruitment of new graduates directly into the perioperative setting.}

Most undergraduate perioperative courses are designed to include both lecture and clinical hours that are spent in different perioperative areas, such as the preoperative unit, OR, and PACU. Students in these courses provided feedback indicating that the experiences were beneficial; however, findings relay that the effect of these experiences on career choices is mixed. Although some results show that partnerships between nursing schools and health care organizations were effective in reducing local nursing shortages, ${ }^{7,14}$ other results indicate that a perioperative experience, although valued by students, is not sufficient to motivate most students to select perioperative nursing as a career immediately after graduation., ${ }^{5,15,16}$ No studies were found in the literature that examine the long-term effect of perioperative courses on career choices. Because some nursing students have indicated that they are more open to considering perioperative nursing in the future 
because of undergraduate experiences, ${ }^{5,8}$ additional research is needed regarding the effectiveness of perioperative courses on career choices over time.

\section{METHODS}

We designed a descriptive study to survey the career choices of nurses four to nine years after graduating from a baccalaureate program that included a perioperative nursing elective. We selected a descriptive survey design to obtain information about positions the nurses held because it is an efficient and cost-effective approach to obtaining the type of data sought for this research. This study is a follow-up to our previous qualitative study conducted when these nurses were students. ${ }^{5}$

\section{Protection of Participants' Rights}

We obtained approval for this study from the university institutional review board. Consent forms were not necessary because the study received approval as an exempt review. At the top of the survey was a statement indicating to participants that returning the survey served as implied consent. Participation was voluntary and we did not request any identifying information. Although the researchers knew who the potential participants were, the researchers took particular care to maintain anonymity by using prepaid return envelopes, thus avoiding the need for return addresses. Despite these precautions, some participants told us their names and wrote personal greetings on their surveys. Only the researchers opened the envelopes, and surveys were kept in a locked drawer. Data were maintained on password-protected computers. As an incentive and token of appreciation, we included a notepad with the college logo on it with the surveys; however, we did not view this incentive as coercive.

\section{Participant Recruitment}

In February of 2016, we contacted all nurses who graduated from one private university in the Midwest and completed a perioperative nursing elective during their junior or senior year. We used a convenience sampling method to generate a list of all individuals who had taken the elective course. Out of 50 names on the list, we were able to obtain the mailing addresses of 49 potential participants and mailed the surveys to the individuals along with prepaid return envelopes. Three of the surveys were returned as undeliverable, but a new address was obtained for one of these and that survey was re-sent. Our final sample consisted of 23 respondents for a return rate of $48.9 \%$. Most surveys were returned within approximately three weeks, but some took more than two months. Because the surveys were anonymous, it was not possible to reach out to individuals who did not return the survey.

\section{Study Procedure}

We created a two-page written survey that could be completed in approximately 10 minutes. It was purposefully brief so that individuals would not feel burdened and would be more likely to participate. The first question asked the participants to list the positions they have held since graduation. Subsequent open-ended questions that we designed to solicit comments about how the perioperative nursing elective influenced their career choices were as follows.

- If you have worked in a perioperative setting, please tell us what influence, if any, the perioperative nursing elective had on your decision to work there.

- How has taking the elective benefited you in your career?

- Would you consider holding a position in perioperative nursing in the future? Why or why not?

Finally, we asked questions regarding demographic data, including gender, race, marital status, and current employment status.

We recorded information about career positions and demographics in a spreadsheet and separately compiled narrative responses from the open-ended questions. As we typed the narrative replies, it was apparent that several ideas were repeatedly expressed. Given the brevity of the responses and the obvious similarities among them, we found that we did not need to develop an in-depth coding method. Instead, we repeatedly read the responses and identified recurring concepts and phrases, and grouped these concepts and phrases to describe key perceptions of the nurses in our sample. At a later time, we reread the original survey responses to be sure that our descriptions were accurate.

\section{Sample Data}

Of the 23 respondents comprising the final sample, there were 21 females (91\%) and two males (9\%). The sample reflected the population on the variable of race: two respondents (9\%) were African American, one (4\%) was 
Hispanic, and the remaining 20 (87\%) were Caucasian. Eleven respondents (48\%) were married and the remaining 12 (52\%) were single. Two (9\%) worked part-time in nursing, two (9\%) worked part-time in a nonnursing position, and the remaining 19 (83\%) worked full-time in nursing. Twelve of the respondents (52\%) indicated that they either were enrolled in a graduate program or had obtained a graduate degree. Of these respondents, most reported that they were enrolled in either a master of science in nursing degree program or a doctor of nursing practice program, although one was enrolled in a joint master of science in nursing and master of business administration program. Five reported having no certifications, and some reported having obtained certifications related to advanced life support or other nursing certifications. Three were certified as advanced practice nurses. One reported having a CRNA, and two others reported having CNOR certifications.

\section{RESULTS}

From the responses to the first question asking participants to describe the influence of the perioperative elective on their decisions to work in the perioperative setting, we discovered that completing the course sensitized them to consider this specialty as a career option. This experience opened doors that allowed them to land their "dream job." We found it interesting that some nurses who had not worked in the perioperative area felt compelled to respond to this first question. There were a few who noted that their career choices have been tangential to perioperative nursing. For example, they indicated that what they learned from the course helped prepare them for caring for surgical patients outside the perioperative setting. Two indicated that although they were offered a position in perioperative nursing, they sought other opportunities.

From the responses to the first question asking participants to describe the influence of the perioperative elective on their decisions to work in the perioperative setting, we discovered that completing the course sensitized them to consider this specialty as a career option.

The responses to our second question asking how the elective had benefited them in their careers were overwhelmingly positive. Individuals who had worked in the perioperative setting indicated that they had a "leg up" over other applicants and benefited because they knew the setting, role, and skills needed to be successful. Participants who had not worked in a perioperative setting expressed that they felt more knowledgeable, confident, and competent because of the course. They indicated that they applied their knowledge and skills to providing preoperative and postoperative nursing care on inpatient units. Furthermore, not only did they believe they were better prepared to educate patients and families about surgery, they recognized that they were sensitized to the surgical patient experience. Of the nurses who were not working in a perioperative setting, 83\% responded that they would consider pursuing such a position in the future because they remained interested in the field. Some indicated that they would move to the perioperative setting except that they were happy where they were currently working. One individual indicated that perioperative nursing was not a fit with her goal to enter graduate school, while another would not consider this option because she found the setting "boring."

Nurses in our sample reported holding 48 nursing positions since graduating (Table 1). Eight of the participants indicated that they had held the same position since graduating, while others indicated that they had held two to four different positions. We noted that perioperative nursing as a specialty represented $10 \%$ of the positions held. One nurse chose the perioperative area as her career specialty and has not worked in any other specialty. Four nurses moved to the perioperative area after working in medicalsurgical nursing positions for approximately two to three years. There was another nurse who became a CRNA after working in an intensive care setting, for a total of six nurses who engaged in the perioperative nursing specialty. We found that the specialty areas that were more popular than perioperative nursing as a career choice were medicalsurgical nursing (35\%) and intensive care nursing (21\%).

\section{DISCUSSION}

We think it is noteworthy that $26 \%$ of the nurses who returned the survey worked in the perioperative specialty. This finding is consistent with Holmes, ${ }^{18}$ who found that $25 \%$ of new graduates pursued perioperative nursing after completing a perioperative elective course. These results support that there is a long-term effect of taking a perioperative nursing elective, and we suggest that such courses may be helpful in addressing the 
Table 1. Career Specialty Choices of Nurses Who Completed the Perioperative Nursing Elective ( $\mathrm{N}=23)$

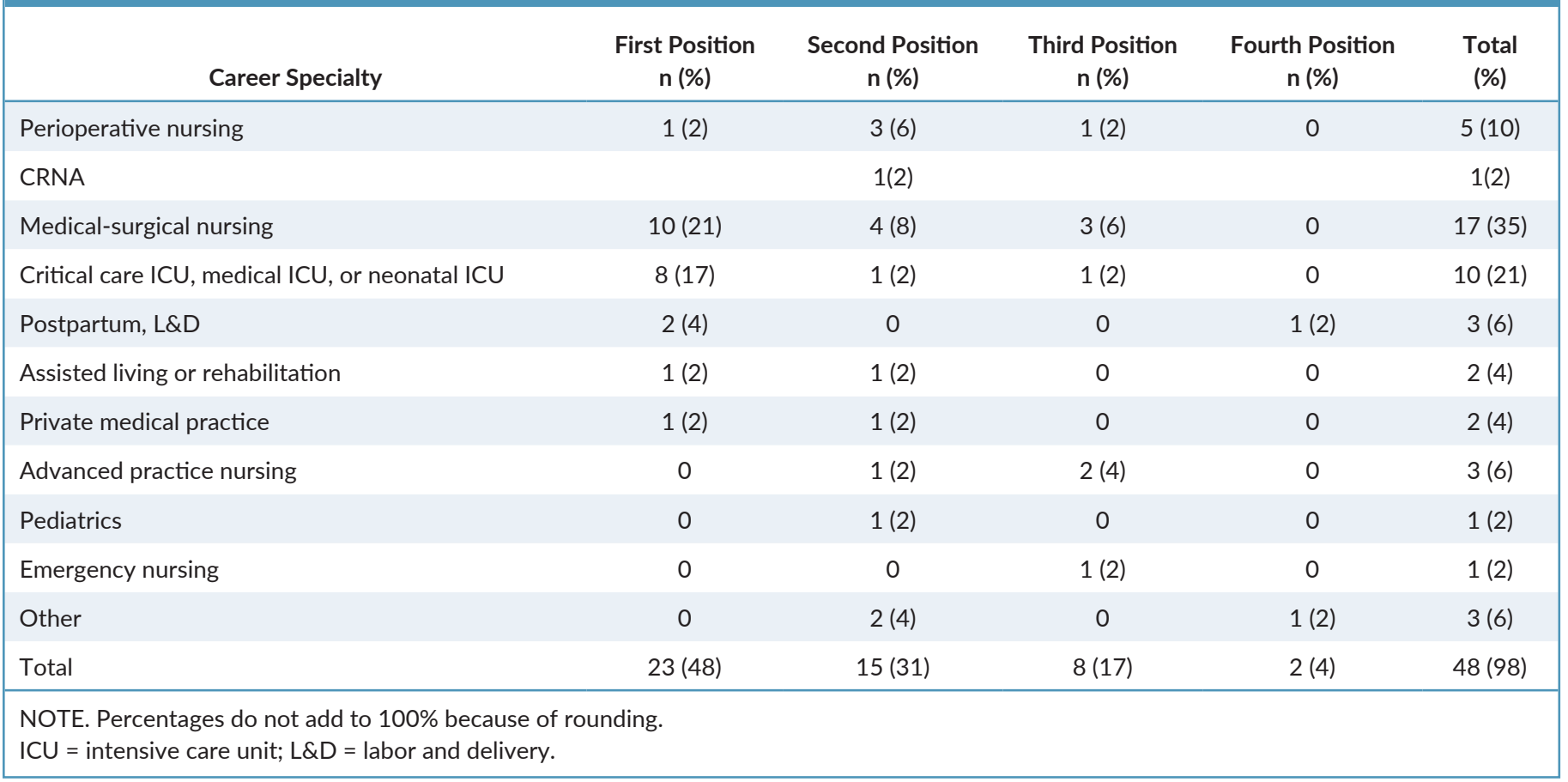

shortage of perioperative nurses. Of the six respondents (five staff RNs and one CRNA) in our study who worked in the perioperative setting, five selected this specialty after working in another area for two to three years. Additionally, the majority indicated that they continued to consider perioperative nursing as a career choice. In our study, one nurse was hired for a perioperative nursing position at the location where he had his clinical experience during the course. This is consistent with findings from other studies, ${ }^{8,11,12,14,15}$ providing additional evidence that academic-practice partnerships are beneficial to both the students and the facilities.

Consideration of career advancement is relevant to the discussion of the perioperative nursing shortage. Of the nurses who were advanced practice nurses, one was a CRNA and another was an acute care nurse practitioner for cardiovascular and thoracic surgery. These nurses indicated that the perioperative nursing elective was helpful in advancing their careers. Additionally, another respondent indicated that she was completing a master's degree to become a perioperative nurse manager. However, other respondents indicated that their career goals for advancement could not be met in the perioperative setting. In our previous study, ${ }^{5}$ we found that students enrolled in a perioperative nursing elective believed that there were limited opportunities for advancement in the perioperative field. Our findings suggest that it is critical for nurse leaders to develop advancement pathways at their facilities to attract and retain nurses in the perioperative setting.

A few nurses who participated in our study expressed reluctance to choose perioperative nursing because of the physical environment, which is a finding consistent with the literature. ${ }^{15}$ The perioperative physical environment is of concern because individuals feel they are being held captive as a result of the lack of windows, the inability to leave the unit, or the lack of break time. ${ }^{5}$ Designing surgical units and creating policies and models that avoid isolation may help attract nurses. For example, an innovative scheduling model that rotates nurses between the perioperative and medical-surgical areas could appeal to nurses. This model may reduce the sense of isolation in the perioperative area and provide a variety of experiences that some nurses desire.

All respondents affirmed that taking the elective course was beneficial regardless of whether they selected perioperative nursing as a career option. They indicated that they were more confident and knowledgeable nurses because of the course. Additionally, our respondents expressed that they were better prepared to perform select skills (eg, maintaining sterile technique, catheter insertion, dressing wounds) and teach patients and families about surgical procedures. Participation in the course afforded them a more holistic understanding of the surgical experience. 


\section{Key Takeaways}

- Most undergraduate nursing programs do not include perioperative content as part of their curricula. A lack of exposure to the specialty may be a contributing factor to perioperative nurse shortages.

- Twenty-three nurses who took a perioperative nursing elective as baccalaureate students responded to a written survey of their career choices since graduating four to nine years ago. Overall, the nurses have held 48 positions since graduating, $10 \%$ of which were in perioperative nursing.

- Responses to the survey indicated a positive outlook and appreciation for the perioperative nursing elective. Nurses who have not worked in the perioperative area commented that they learned a lot from the course and continued to consider perioperative nursing as a potential future specialty.

- Offering a perioperative nursing elective may affect career choices in the future and increase the number of nursing students who choose perioperative nursing as a specialty.

These benefits were noted most frequently by nurses who worked in medical-surgical and obstetric units.

Even though most nurses in our sample elected not to work in a perioperative setting, they remained open to doing so in the future. However, some respondents indicated that they "loved" where they currently worked; thus they were not considering a career change at the time of the survey. It seems to us that nurses become comfortable and "happy" with their positions, which may make it more difficult for them to change to the perioperative area later in their career. Perioperative nurse managers should move away from the philosophy of requiring medical-surgical experience before they hire nurses. We believe that nurses need to be drawn to the perioperative track early in their careers before they become comfortable in other nursing specialties. It is wise to capitalize on the enthusiasm new nursing graduates have about perioperative nursing by hiring them immediately after graduation; otherwise, there is a risk that they will become comfortable in another area and not enter perioperative nursing.

It is evident that an elective perioperative course can influence career choices. ${ }^{5,7,8,14-18}$ Offering an elective is worthwhile, and nurse educators have a responsibility to create meaningful student experiences in perioperative nursing. For existing courses, we believe that educators need to embrace collaborative partnerships with perioperative staff members so that positive and engaging student clinical experiences can be created in sufficient numbers. It may not be practical for every nursing program to require all students to take a course about perioperative nursing. In lieu of clinical placements in the OR, simulation experiences may offer a viable alternative. For example, interdisciplinary OR simulations could provide students in medical-surgical courses rich opportunities for learning content, mastering skills, and practicing roles related to perioperative nursing. The knowledge, skills, and attitudes gained will serve to grow their confidence and can be applied to other specialty areas.

\section{Limitations}

Although our findings suggest that the perioperative elective course may have influenced nursing career choice, caution is warranted because of the study design. We did not test the instrument for validity or reliability, and therefore the questions could have been misinterpreted by respondents. However, their responses were consistent with the intention of the questions and it was a simple survey with a table for listing nursing positions held since graduation followed by some open-ended questions. Another limitation is possible bias in our questions. We assumed a positive effect from the course because in our previous qualitative study, we found that taking the elective was beneficial. ${ }^{5}$ Selection bias is a threat because participants who did not return the survey were more likely to have not worked in a perioperative area. Furthermore, students who completed the elective may have had greater interest in perioperative nursing compared with students who did not enroll in the elective. Additionally, there may have been bias in work experiences related to taking the perioperative course. For example, managers may have been more likely to hire these new graduates rather than other applicants who had not had this experience.

\section{CONCLUSION}

Strategies for attracting nurses to the perioperative area are needed to address a growing nursing shortage in this 
specialty. The purpose of our descriptive survey was to determine if an undergraduate perioperative nursing elective influenced career choices of nurses four to nine years after graduating. Our study is pertinent because no follow-up studies have been reported in the literature regarding career choices after taking a perioperative nursing elective. We found that such a course may be beneficial in attracting nursing students to the perioperative field. The nurse participants reported that taking the elective was a factor in their hire into a position. The nurses perceived that taking the course enhanced their knowledge, skills, and confidence. Even four to nine years after graduation, our respondents continued to be interested in pursuing a career in perioperative nursing in the future. We found it remarkable that after such a long time, nurses who completed a perioperative nursing elective continue to value what they learned and perceive that the course continues to influence their career choices. Because of these perceptions, we advocate that investing in an elective course on perioperative nursing is worthwhile.

Editor's notes: Periop 101 is a trademark of AORN, Inc, Denver, CO. CNOR is a registered trademark of the Competency \& Credentialing Institute, Denver, CO.

\section{REFERENCES}

1. Young R, Takahashi J, Cheney A. Project alpha goes into action. AORN J. 1981;34(5):920-939.

2. McNamara SA. Perioperative nursing in nursing school curricula. AORN J. 2006;83(2):301-304.

3. Sherman RO. Why we should be concerned about a shortage of perioperative nurses. http://www.emerg ingrnleader.com/periope/. Published October 25, 2012. Accessed August 24, 2018.

4. Sherman RO, Patterson P, Avitable T, Dahle J. Perioperative nurse leader perspectives on succession planning: a call to action. Nurs Econ. 2014;32(4):186-203.

5. Schmidt NA, Brown JM, Holmes L. Student perceptions about the influence of a perioperative nursing elective on career preferences. Int J Nurs Educ Scholarsh. 2016;13(1):55-62.

6. Mitchell L, Stevens D, Goodman J, Brown M. Establishing a collaborative relationship with a college of nursing. AORN J. 2002;76(5):842-850.

7. Ball K, Doyle D, Oocumma NI. Nursing shortages in the OR: solutions for new models of education. AORN J. 2015;101(1):115-136.
8. Tschirch P, Leyden K, Dufrene C, Land S. Introducing perioperative nursing as a foundation for clinical practice. AORN J. 2017;106(2):121-127.

9. Byrne $M$, Root S, Culbertson L. Integrating perioperative content in nursing curricula: a case study approach. AORN J. 2016;103(6):597-604.

10. Mott J. Implementation of an intraoperative clinical experience for senior level baccalaureate nursing students. AORN J. 2012;95(4):445-452.

11. Chappy S, Madigan PD, Doyle DS, Conradt LA, Tapio NC. Preparing the next generation of perioperative nurses. AORN J. 2016;103(1):104.e1-104.e7. https:// doi.org/10.1016/j.aorn.2015.11.016.

12. Penprase B, Monahan J, Poly-Droulard L, Prechowski S. Student immersion in perioperative nursing. AORN J. 2016;103(2):189-197.

13. Castelluccio D. Educating for the future. AORN J. 2012;95(4):482-491.

14. Gregory S, Bolling DR, Langston NF. Partnerships and new learning models to create the future perioperative nursing workforce. AORN J. 2014;99(1):96-105.

15. Messina BAM, lanniciello JM, Escallier LA. Opening the doors to the OR: providing students with perioperative clinical experiences. AORN J. 2011;94(2):180-188.

16. Foran P. Effects of guided undergraduate perioperative education on recruiting novice RNs and retaining experienced RNs. AORN J. 2015;102(3):254-261.

17. Foran P. Undergraduate surgical nursing preparation and guided operating room experience: a quantitative analysis. Nurse Educ Pract. 2016;16(1):217-224.

18. Holmes SP. Implementing a perioperative nursing elective in a baccalaureate curriculum. AORN J. 2004;80(5):902-910.

Nola A. Schmidt, PhD, RN, CNE, is a professor at Valparaiso University College of Nursing and Health Professions, IN. Dr Schmidt has no declared affiliation that could be perceived as posing a potential conflict of interest in the publication of this article.

Janet M. Brown, PhD, RN, was a dean and professor at Valparaiso University College of Nursing and Health Professions, IN, at the time this article was written. Dr Brown has no declared affiliation that could be perceived as posing a potential conflict of interest in the publication of this article. 